\title{
ANÁLISE DO IMPACTO DAS TÉCNICAS ANTI-FORENSE E HARDENING NO SISTEMA OPERACIONAL WINDOWS 10 EM UMA ANÁLISE FORENSE, OBJETIVANDO O AUMENTO DE SEGURANÇA EM NOTEBOOKS
}

\author{
Lucas Maurício Alves Zigunow, Georges Daniel Amvame-Nze, Rafael Timóteo de Sousa Júnior \\ e William Ferreira Giozza \\ Programa de Pós-Graduação Profissional em Engenharia Elétrica (PPEE), Departamento de Engenharia Elétrica \\ Faculdade de Tecnologia, Universidade de Brasília, Brasil
}

\begin{abstract}
RESUMO
Nos dias atuais, a segurança da informação tem sido abordada cada vez mais nos meios acadêmicos e civis, objetivando à proteção de dados contra ataques cibernéticos realizados explorando vulnerabilidades encontradas em sistemas operacionais ou em aplicações, configurações mal feitas ou até ações tomadas por usuários que possam permitir a um invasor obter acesso e controle de um computador. Poucos assuntos abordam e definem métodos que possam ser utilizados para proteger computadores e os dados armazenados em disco e memória em caso de perda ou roubo. Visando o aumento da segurança nos computadores contra acessos indevidos, neste trabalho serão utilizadas de maneira conjugada e especializada, técnicas anti-forense e hardening do sistema operacional Microsoft Windows 10 com intuito de aumentar a segurança dos computadores.
\end{abstract}

\section{PALAVRAS-CHAVE}

Segurança de Computadores, Anti-Forense Computacional, Hardening de Sistemas Operacionais Windows 10, Proteção de Computadores

\section{INTRODUÇÃO}

Devido ao crescente número de ataques cibernéticos, as empresas e órgãos governamentais estão investindo maior quantidade de recursos na proteção dos seus sistemas e serviços, criando desde políticas e normas para seus funcionários voltadas para a segurança da informação, até a implementação de dispositivos para monitorar, defender e proteger seus ativos de TI contra os ataques cibernéticos.

Algumas políticas de segurança da informação, voltadas para o usuário final abordam algumas questões que são principalmente direcionadas para a navegação na Internet, pois é onde atualmente ocorrem os principais casos de ataques. No Brasil, um exemplo é a Cartilha de Segurança para a Internet publicada pelo CERT.br, que apresenta ações a serem tomadas por uma pessoa, principalmente para realizar a utilização da Internet, de forma mais segura.

Quanto à proteção voltada especificamente aos dispositivos, poucos assuntos são abordados. Em resumo, os principais temas relacionados a isso são: instalar software apenas de fontes conhecidas e originais, realizar a atualização de softwares e sistema operacional, instalação de software de antivírus, não permitir que o computador acesse o sistema operacional diretamente (ou seja, utilizar senha para acessar o sistema operacional) e utilizar criptografia nos discos. Ou seja, mais de cunho procedimental de usuários do que de configuração de hardware.

Caso um computador seja roubado ou perdido, quão seguros estarão os dados armazenados nesse dispositivo que esteja utilizando essas medidas apresentadas? Outro exemplo de ameaça é que atualmente, ao entrar em outro país, os agentes que trabalham nas alfândegas estão solicitando vistoriar equipamentos eletrônicos, inclusive acessando e analisando o conteúdo dos dados neles armazenados, em busca de dados ilícitos. Nessas vistorias, algum arquivo pode ser copiado e/ou apagado, além de também poder ser instalada alguma aplicação maliciosa durante a inspeção. 
Com o objetivo de aumentar a segurança nos computadores, este trabalho pretende com o auxílio das técnicas de anti-forense e de hardening no sistema operacional Windows 10, demonstrar as medidas a serem tomadas e como aferi-las através da realização de uma análise forense nos notebooks utilizados nesse trabalho.

\section{CONCEITUAÇÃO DOS TEMAS ABORDADOS}

As técnicas de anti-forense computacional são utilizadas de forma intencional ou acidental para obscurecer, encriptar ou esconder dados de ferramentas forenses (PHILIPP et al., 2010). O hardening do sistema operacional é um conjunto de técnicas que visa reduzir a superfície de ataque, retirando diversas permissões e configurações do sistema operacional implementadas como configuração padrão (BELDING, 2019). A perícia forense computacional envolve a obtenção e a análise de informações digitais para serem usadas como evidência em casos criminais, civis e administrativos (NELSON et al., 2010).

Neste trabalho, hipoteticamente assumimos que uma pessoa tenha perdido seu notebook, e que nesse caso a perícia forense computacional será a atuação de uma pessoa que tenha encontrado o notebook e esteja tentando obter os dados nele contidos. As técnicas de hardening e de anti-forense computacional implementadas visam proteger os dados ali presentes. Essa hipótese tem como objetivo avaliar os impactos das técnicas que serão abordadas em uma análise forense.

$\mathrm{O}$ aprimoramento da segurança dos computadores será a união das técnicas anti-forense computacional e hardening do sistema operacional visando proteger o computador contra acessos indevidos, buscando a proteção dos dados e do sistema. É importante ressaltar que essas técnicas não estão sendo demonstradas com intuito de cobrir qualquer traço ou vestígio de crimes cibernéticos.

\subsection{Implementações de Segurança na Inicialização do Notebook}

O primeiro tema a ser tratado visa utilizar medidas de segurança na inicialização do notebook. Utilizaremos apenas equipamentos que possuam BIOS Unified Extensible Firmware Interface (UEFI), devido a ter algumas configurações que o padrão de BIOS antigo não possuía.

Algumas configurações da UEFI dependerão do software que gerencia a própria UEFI, que pode ou não conter alguns recursos. Em alguns casos, atualizar a firmware do UEFI pode trazer alguns novos recursos que não estavam habilitados, além de corrigir alguma falha de segurança que possa existir em versões anteriores.

Também é possível habilitar dois recursos importantes para a proteção do computador, o Secure Boot e o Trusted Platform Module (TPM). O TPM é um recurso que não está disponível em todos os computadores, pois depende de ter um chip instalado na placa-mãe usada pelo computador.

O Secure Boot é uma função que na configuração e montagem do computador pela fábrica, a UEFI cria uma lista de chaves para identificar componentes de hardware, firmware e códigos de carregamento dos sistemas operacionais confiáveis. Essas listas visam proteger o computador de malwares ou que o computador não inicialize utilizando mídias ilegais ou por sistemas operacionais não identificados na lista de chaves (KHANSE, 2017).

O TPM é um chip projetado para que a inicialização do sistema seja mais segura e para desempenhar as funções criptográficas, realizando medição de integridade do sistema, além da criação e gerência de uso das chaves criptográficas (MICROSOFT DOCS, 2018).

É importante salientar que algumas dessas configurações podem ser desfeitas, como por exemplo, definir o acesso ao UEFI mediante a senha, inicializar o sistema operacional utilizando uma senha ou impedir o uso de alguma mídia de CD-ROM ou pen drive contendo outro sistema operacional. Tais alterações podem simplesmente serem desfeitas alterando alguns pinos na placa-mãe ou retirando a bateria que mantém as informações da UEFI gravadas.

As medidas adotadas para a proteção da UEFI, devido à facilidade de serem desfeitas, tem como principal objetivo afastar pessoas de baixo conhecimento em computação ou que não tenham como obter acesso aos componentes físicos do notebook, a fim de serem impedidas de obter acesso ao sistema operacional e aos dados nele contidos. 


\subsection{Segurança dos Discos Rígidos e dos Dados Armazenados}

Após trabalhar na inicialização do equipamento a ser utilizado, o próximo componente a ter implementadas camadas extras de segurança é o disco rígido ou hard disk (HD). A melhor forma de proteger o HD é a utilização de criptografia, pois com ela é possível criptografar o disco inteiro, ou uma partição, ou criar um contêiner para armazenar arquivos. Utilizando a criptografia de disco, também é possível criar um segundo sistema operacional, o qual será utilizado apenas para armazenar os dados mais sensíveis.

As seguintes técnicas anti-forense serão utilizadas para armazenar os arquivos no HD de forma segura e dificultar o acesso aos dados mais sensíveis: armazenar arquivos no slack space, armazenar arquivos no NTFS Master File Table (MFT), armazenar arquivos nos bad blocks e armazenar arquivos usando os espaços reservados do inode.

O slack space é um espaço não utilizado dentro de um cluster do disco rígido. O MFT é uma estrutura que armazena os dados referentes à localização de todos os arquivos e diretórios dentro de um disco rígido. Os bad blocks são defeitos físicos na superfície de um disco rígido, e que nesse caso será forjado e utilizado para esconder arquivos. O inode, no caso do sistema de arquivo NTFS File Index Number, contém diversas informações de um arquivo ou pasta, como o usuário dono do arquivo, permissão de acesso ou informações sobre data e hora de criação do arquivo ou de modificação.

\subsection{Hardening no Sistema Operacional Windows 10}

As versões Home e Professional do sistema operacional Windows 10 são mais encontradas em notebooks. A versão Home vem instalada principalmente em notebooks comprados para o uso pessoal, enquanto a versão Professional é encontrada em notebooks utilizados por empresas, pois possui mais opções que a versão Home.

As implementações de seguranças, através do hardening, visam diminuir as superfícies de ataques que podem ser encontradas no sistema operacional Windows 10, utilizando serviços e configurações que são habilitados por padrão e que podem ser desabilitados.

Serão realizadas diversas configurações que alterarão como exemplo: política local do computador, configuração de usuário, modificações no registro do Windows e ajustes no sistema operacional.

\section{METODOLOGIA APLICADA AO TRABALHO EM ANDAMENTO}

Utilizando as técnicas conceituadas anteriormente, serão implementadas medidas para aumentar a segurança visando dificultar o acesso indevido ao dispositivo e aos dados armazenados, através de técnicas utilizadas contra uma análise forense e também utilizando técnicas de hardening do sistema operacional Windows 10.

Para alcançar esses objetivos, inicialmente foi realizado um estudo bibliográfico sobre os temas, visando encontrar os principais pontos a serem trabalhados. Dessa forma, encontramos os seguintes assuntos, proteção UEFI, proteção dos discos rígidos e hardening do sistema operacional Windows 10.

Estudando cada tema individualmente, começando pela UEFI, observamos que existem itens que podem ser utilizados para ampliar a segurança, como o uso de senha tanto para acessar a UEFI quanto para inicializar o sistema operacional, a habilitação caso o notebook possua o chip TPM, configurar a função de secure boot e impedir que sejam utilizadas mídias com outros sistemas operacionais para acessar o computador.

$\mathrm{Na}$ aplicação de segurança nos discos rígidos, serão explorados assuntos que visam garantir tanto a segurança física quanto dos dados armazenados no dispositivo. Quanto à segurança física dos discos, abordaremos assuntos como por exemplo a utilização de ATA Password, que tem por objetivo impedir que seja feito um clone do disco rígido por duplicadores forenses. Em relação à proteção lógica, o uso de criptografia nos discos visa impedir o acesso aos seus dados, bem como a criação de um contêiner com outro sistema operacional, que pode ser usado para trabalhar dados sensíveis.

Após realizarmos a segurança da UEFI e do disco rígido, o próximo passo é realizar o hardening no sistema operacional Windows 10. Um exemplo disso é remover os privilégios de administrador de todos os usuários, criando um perfil de administração que será utilizado apenas quando necessário. Outro exemplo é impedir que o sistema operacional, ao ser desligado, grave nos arquivos pagefile.sys e hiberfil.sys o que está sendo executado na memória para agilizar o processo de inicialização do sistema operacional, pois nesses dois arquivos é possível extrair informações como senhas em claro. 
Após as implementações das técnicas citadas e visando validar e demonstrar os impactos que possam causar, definir o nível de proteção que essas implementações podem oferecer no tocante à proteção do computador bem como dos dados ali presentes, será realizada uma análise forense nos dispositivos, objetivando ganhar o acesso ao equipamento e obter o máximo de informações que possam ser extraídas.

Essa análise forense será realizada de forma separada em cada um dos temas trabalhados, com o objetivo de demonstrar a efetividade de cada um deles quanto ao ganho de segurança no computador. Posteriormente será realizada uma análise forense com todos os temas aplicados em conjunto, objetivando avaliar de maneira geral a eficiência da combinação dessas medidas propostas para aumentar a segurança dos computadores.

Para realizar a validação das técnicas descritas neste artigo, serão aplicadas em três notebooks de marca, modelo e sistemas operacionais com versões diferentes com o objetivo de ampliar a variedade de objetos a serem testados.

A variação das marcas e modelos dos equipamentos utilizados foram definidos por possuírem configurações variadas de hardware e software, como um exemplo a BIOS, que dependendo da marca e modelo, possuí ou não algum recurso habilitado que pode ser utilizado.

\section{CONSIDERAÇÕES FINAIS}

Este trabalho visa criar uma metodologia que possa ser usada por todos a fim de aumentar a segurança nos equipamentos de informática do tipo notebook, contra acessos indevidos e tentando mitigar o vazamento de dados e informações que possam causar algum prejuízo para as pessoas ou empresas.

Outro objetivo deste trabalho é medir o impacto das técnicas de hardening no sistema operacional Windows 10 em uma análise forense, tentando descobrir alguma evidência, encontrar arquivos protegidos e/ou obter alguma informação que possa ser recuperada.

Além disso, este trabalho será submetido para apreciação da Força Aérea Brasileira (FAB), visando criar uma normatização para a proteção dos computadores (notebooks) utilizados pelos militares e civis que trabalhem naquele órgão e que estejam realizando missões externas às unidades militares a que pertençam, visando aumentar a segurança dos equipamentos e dos dados neles contidos.

\section{REFERÊNCIAS}

Belding, Greg (2019). Windows 10 Hardening Techniques. [online]. Disponível em: https://resources.infosecinstitute.com/category/certifications-training/securing-windows-ten/windows-10-hardeningtechniques/ [Acessado em: 22 de Outubro de 2019].

Hoffman, Chris. How Secure Boot Works on Windows 8 and 10, and What It Means for Linux. [online]. (Última atualização 5 de Julho de 2017). Disponível em: https://www.howtogeek.com/116569/htg-explains-how-windows-8s-secure-bootfeature-works-what-it-means-for-linux/ [Acessado em: 21 de Outubro de 2019].

Hoffman, Chris. How to Secure Your Computer With a BIOS or UEFI Password. [online]. (Última atualização 12 de Julho de 2017). Disponível em: https://www.howtogeek.com/186235/how-to-secure-your-computer-with-a-bios-or-uefipassword/ [Acessado em: 21 de Outubro de 2019].

Khanse, Anand (2017). How to Secure the Windows 10 Boot Process. [online]. Disponível em: https:/www.thewindowsclub.com/secure-windows-10-boot-process [Acessado em: 21 de Outubro de 2019].

Microsoft Docs (2018). Visão geral da tecnologia Trusted Platform Module. [online]. Disponível em: https://docs.microsoft.com/pt-br/windows/security/information-protection/tpm/trusted-platform-module-overview [Acessado em: 19 de Outubro de 2019].

Nelson, Bill. et al, 2010. Guide to computer Forensics and Investigations. 4th ed. Course Technology - CENGAGE Learning, Boston, Massachusetts, USA.

Philipp, Aaron. et al, 2010. Hacking Exposed Computer Forensics, 2nd ed. McGraw-Hill, New York, New York, USA.

Schneider, Michael (2017). Windows 10 Hardening. [online]. (Última atualização 2 de Setembro de 2019). Disponível em: https://github.com/0x6d69636b/windows_hardening [Acessado em: 21 de Outubro de 2019]. 